ARTICLE OPEN

There are amendments to this paper

\title{
Deep learning algorithm predicts diabetic retinopathy progression in individual patients
}

\author{
Filippo Arcadu ${ }^{1,2}$, Fethallah Benmansour ${ }^{1,2}$, Andreas Maunz ${ }^{1,2}$, Jeff Willis ${ }^{3,4}$, Zdenka Haskova ${ }^{3,4,7 凶}$ and Marco Prunotto $\mathbb{D}^{2,5,6,7 凶}$
}

The global burden of diabetic retinopathy (DR) continues to worsen and DR remains a leading cause of vision loss worldwide. Here, we describe an algorithm to predict DR progression by means of deep learning (DL), using as input color fundus photographs (CFPs) acquired at a single visit from a patient with DR. The proposed DL models were designed to predict future DR progression, defined as 2-step worsening on the Early Treatment Diabetic Retinopathy Diabetic Retinopathy Severity Scale, and were trained against DR severity scores assessed after 6, 12, and 24 months from the baseline visit by masked, well-trained, human reading center graders. The performance of one of these models (prediction at month 12) resulted in an area under the curve equal to 0.79 . Interestingly, our results highlight the importance of the predictive signal located in the peripheral retinal fields, not routinely collected for DR assessments, and the importance of microvascular abnormalities. Our findings show the feasibility of predicting future DR progression by leveraging CFPs of a patient acquired at a single visit. Upon further development on larger and more diverse datasets, such an algorithm could enable early diagnosis and referral to a retina specialist for more frequent monitoring and even consideration of early intervention. Moreover, it could also improve patient recruitment for clinical trials targeting DR.

npj Digital Medicine (2019)2:92; https://doi.org/10.1038/s41746-019-0172-3

\section{INTRODUCTION}

Vision loss due to diabetic eye disease is on the rise and it is expected to reach epidemic proportions globally in the next few decades. In 2017, 425 million people worldwide had diabetes, and this number is estimated to increase to 642 million by $2040{ }^{1}$ Diabetic retinopathy (DR) is the most common and insidious microvascular complication of diabetes, and can progress asymptomatically until a sudden loss of vision occurs. Almost all patients with type 1 diabetes mellitus and $\sim 60 \%$ of patients with type 2 diabetes mellitus will develop retinopathy during the first 20 years from onset of diabetes. ${ }^{2}$ However, DR often remains undetected until it progresses to an advanced vision-threatening stage. The current state of DR screening in the real world, based on assessment of color fundus photographs (CFPs, see Fig.1a) by a retina specialist or a trained grader, leaves a large proportion of patients undiagnosed and therefore receiving medical help too late, in part due to low adherence and access to retina screening visits. ${ }^{3,4}$ In-person expert examinations are impractical and unsustainable given the pandemic size of the diabetic population. $^{5-7}$ Notwithstanding, early detection and prevention of DR progression are essential to mitigate the rising threat of DR.

Artificial intelligence (AI) may offer a solution to this conundrum. Deep learning (DL), and specifically, deep convolutional neural networks (DCNNs), ${ }^{8}$ can be used for an end-to-end assessment of raw medical images to produce a target outcome prediction. The diagnostic use of DCCN algorithms is already spreading in various healthcare areas, ${ }^{9,10}$ such as radiology, ${ }_{1}^{11,12}$ dermatology, ${ }^{13}$ and pathology. ${ }^{14}$ In ophthalmology, groundbreaking work has recently been conducted on the automation of DR grading $^{15-17}$ and prediction of cardiovascular risk factors ${ }^{18}$ by DCNN analysis of CFPs.
The purpose of this work was to go beyond the use of DL for DR diagnostics ${ }^{15-17,19}$ and to assess the feasibility of developing DCNNs operating on 7-field CFPs that can predict the future threat of significant DR worsening at a patient level over a span of 2 years after the baseline visit.

To achieve that, our DCNNs have been trained on high-quality 7-field CFPs acquired at a single visit and graded for DR severity by masked and well-trained reading center experts, using the validated Early Treatment Diabetic Retinopathy Study (ETDRS) Diabetic Retinopathy Severity Scale (DRSS) ${ }^{20}$ from large controlled clinical trials. Previous studies have limited the deployment of DCNNs to fovea- or optic nerve-centered CFPs. ${ }^{15-19}$ Our findings highlight the importance of the predictive signal located in the peripheral retinal fields of patients with DR and suggest that such a predictive algorithm, upon further development and proper validation, could help fight blindness by identifying fast DR progressors for referral to a retina specialist or inclusion in clinical trials targeting early stages of DR.

\section{RESULTS}

Model performance

The DL models (the architecture is shown in Fig. 1c and described in detail in the Methods section) were able to predict 2-step or more ETDRS DRSS worsening at 6, 12, and 24 months with an area under the curve (AUC) of $0.68 \pm 0.13$ (sensitivity, $66 \% \pm 23 \%$; specificity, $77 \% \pm 12 \%$ ), $0.79 \pm 0.05$ (sensitivity, $91 \% \pm 8 \%$; specificity, $65 \% \pm 12 \%$ ), and $0.77 \pm 0.04$ (sensitivity, $79 \% \pm 12 \%$; specificity, $72 \% \pm 14 \%)$ respectively. The receiver operating characteristic curves of the fivefold cross-validation (CV) are shown in Fig. 2. By comparing these values with the average AUC

\footnotetext{
${ }^{1}$ Roche Informatics, Roche, Basel, Switzerland. ${ }^{2}$ Roche Personalized Healthcare, Roche, Basel, Switzerland. ${ }^{3}$ Clinical Science Ophthalmology, Genentech, Inc., South San Francisco, CA, USA. ${ }^{4}$ Roche Personalized Healthcare, Genentech, Inc., South San Francisco, CA, USA. ${ }^{5}$ Immunology, Infectious Disease \& Ophthalmology, Roche, Basel, Switzerland. ${ }^{6}$ School of

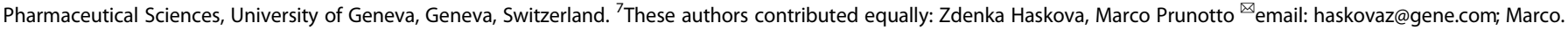
Prunotto@roche.com
} 


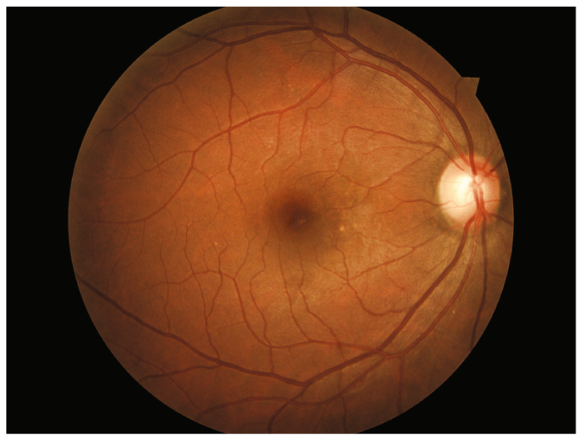

Patient with DR

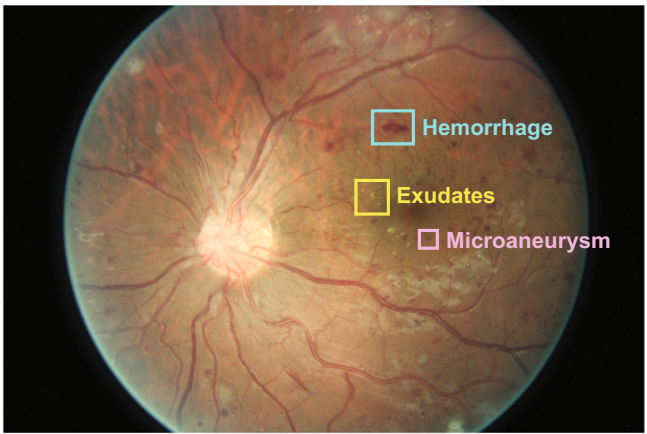

b
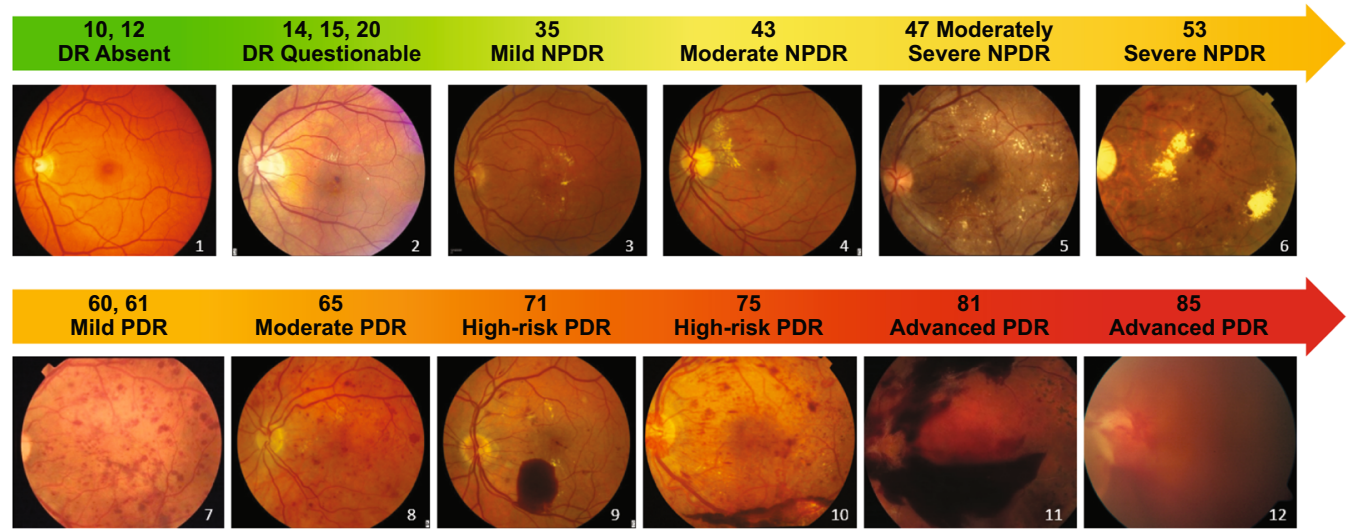

C

+2 DRSS at month X?

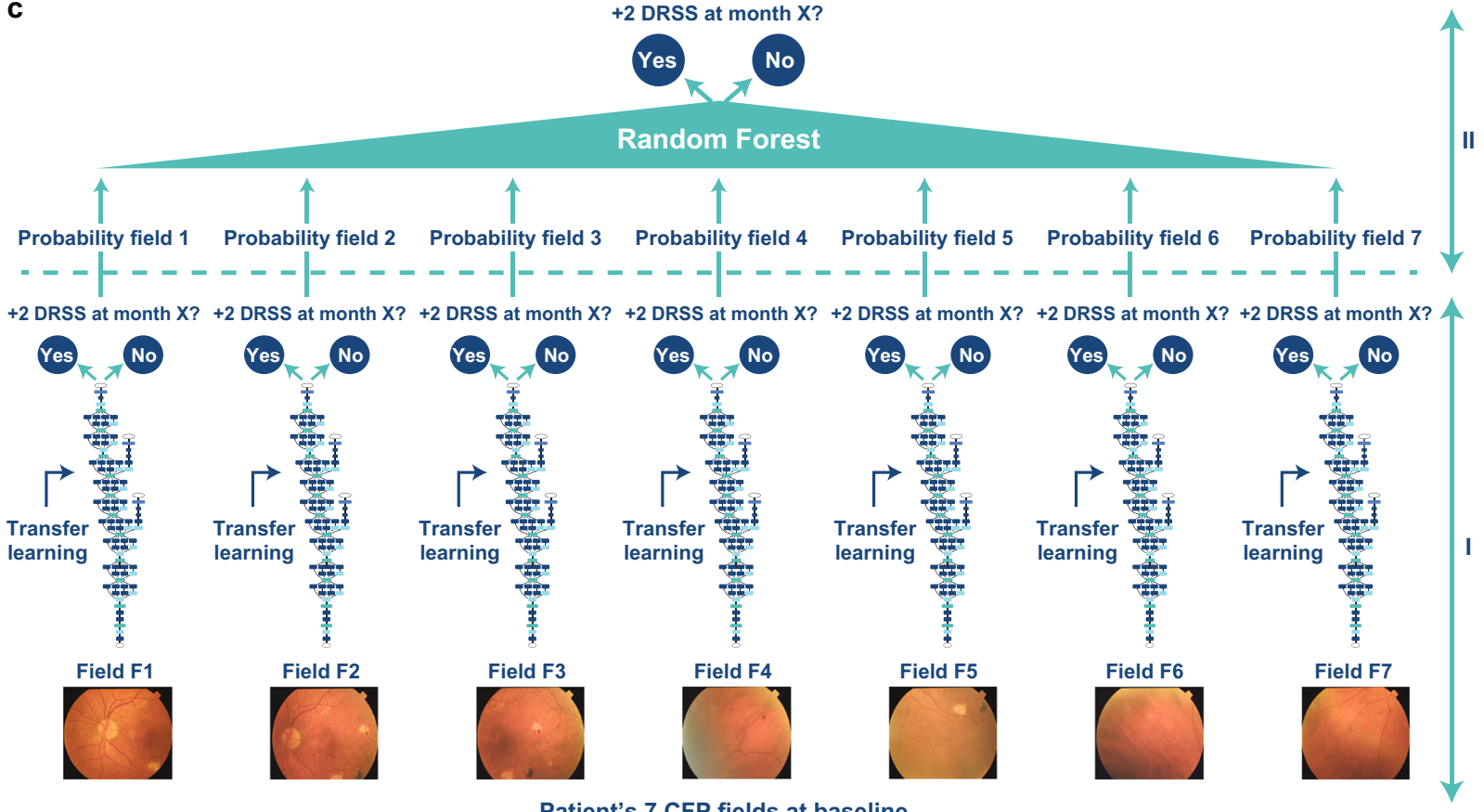

Patient's 7 CFP fields at baseline

Fig. 1 An overview of retinal imaging features analyzed to assess diabetic retinopathy (DR) severity and a schematic of the study design. a Example of fovea-centered color fundus photographs (CFPs) of a patient without DR (left) and a patient with signs of DR (right). In the CFP of the patient with signs of DR (right), one example each of hemorrhage, exudate, and a microaneurysm are highlighted. Both examples have been selected from the Kaggle DR dataset. ${ }^{47}$ b Schematic of the Diabetic Retinopathy Severity Scale (DRSS) established by the Early Treatment Diabetic Retinopathy Study (ETDRS) group to measure DR worsening over time. c Schematic of the two-phase modeling to detect two-step or more DRSS worsening over time. In phase I, field-specific Inception-v3 deep convolutional neural networks (DCNNs) called "field-specific DCNNs" or "pillars" are trained by means of transfer learning to predict whether the patient will progress two ETDRS DRSS steps. In phase II, the probabilities independently generated by the field-specific DCNNs are aggregated by means of random forest 


\section{Month 6}

Five-fold CV separate ROCs

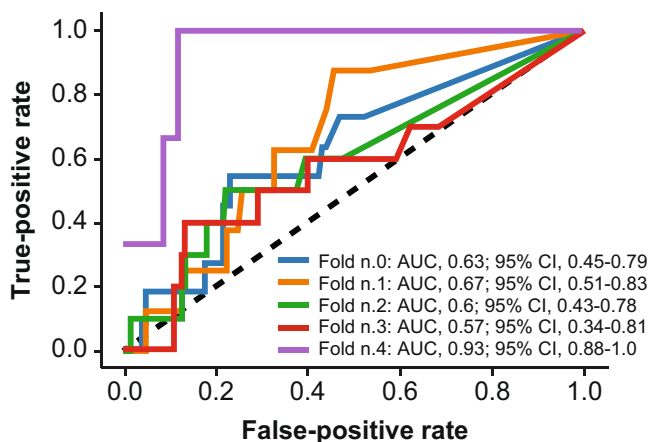

Five-fold CV composite ROC

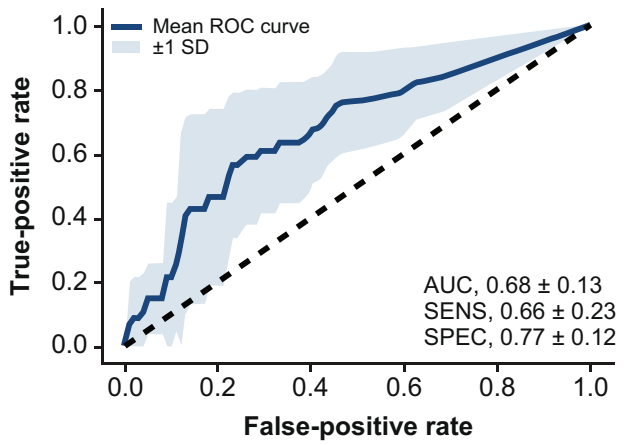

Month 12

Five-fold CV separate ROC

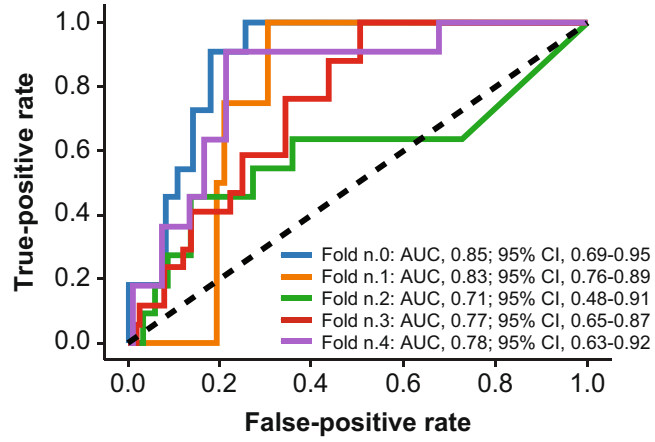

Five-fold CV composite ROC

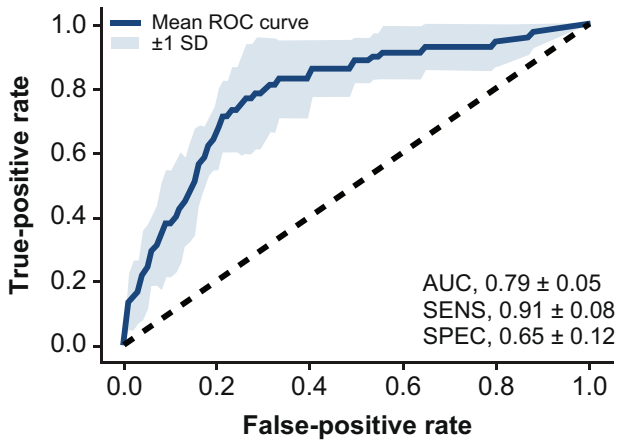

Month 24

Five-fold CV separate ROC

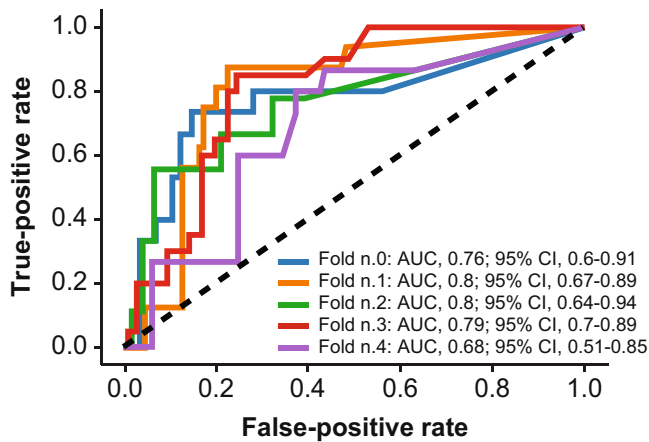

Five-fold CV composite ROC

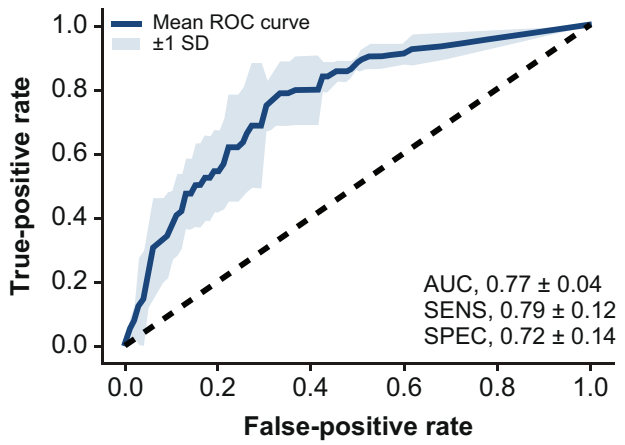

Fig. 2 Summary of the results for the prediction of two-step or more diabetic retinopathy progression at months 6, 12, and 24 using 7-field color fundus photographs of patients at baseline. AUC area under the curve, $\mathrm{Cl}$ confidence interval, CV cross-validation, ROC receiver operating characteristic, SD standard deviation, SENS sensitivity, SPEC specificity

of the individual field-specific DCNN models (Table 1), it appears that the aggregation did significantly improve the overall performance, especially for month $12(P=0.00049)$ and $24(P=$ 0.00042). Results for month 6 were weaker compared with months 12 and 24, mainly due to the scarcity of patients with DR progression within the first 6 months ( $6 \%$; see details in the "Methods" section).

Models using just the ETDRS DRSS grade at baseline achieved an AUC $\sim 0.52$; only slightly above the "tossing-coin line" (Supplementary Fig. 2).

Predictive value of the individual CFP fields

The optic-nerve-centered field (F1) and the fovea-centered field (F2) are generally regarded as the most important fields in a standard ophthalmoscopy exam. The purpose of this analysis was to evaluate the predictive signal of DR progression in these central retinal fields compared with the peripheral fields (F3, F4, F5, F6, and F7). We found that the main predictive contribution came from the peripheral retinal fields (F3, F4, F5, F6, and F7) that encompass areas of the retina far from both the fovea and optic nerve. Performance comparisons between models trained only on central fields (F1 and F2) versus models trained on all seven retinal fields support our finding. For this comparison, we performed the random forest (RF) aggregation only with the probabilities generated by the F1- and F2-specific DCNNs (Fig. 1C). Using this subset of the RF inputs brought the AUC down to $0.62 \pm 0.13$ (from $0.68 \pm 0.13$ with all seven fields; $P=0.0486$ ), $0.64 \pm 0.05$ (from $0.79 \pm 0.05$ with all seven fields; $P=0.00014$ ), and $0.69 \pm 0.05$ 
F. Arcadu et al.

Table 1. Performance of the individual field-specific DCNNs in terms of AUC

\begin{tabular}{|c|c|c|c|c|c|c|c|}
\hline Month & F1 & F2 & F3 & F4 & F5 & F6 & F7 \\
\hline 12 & $0.68 \pm 0.04$ & $0.62 \pm 0.07$ & $0.67 \pm 0.05$ & $0.75 \pm 0.06$ & $0.70 \pm 0.04$ & $0.72 \pm 0.05$ & $0.74 \pm 0.03$ \\
\hline
\end{tabular}

(from $0.77 \pm 0.04$ with all seven fields; $P=0.0023$ ) for months 6,12 , and 24 , respectively.

The analysis by "Shapley Additive Explanations" (SHAP) ${ }^{21}$ allows for interpretation of predictions of complex symbolic machine learning (ML) models by attributing the descriptors to the weights of importance. Here it was used to evaluate the field-specific DCNN contribution to the final RF aggregation. Figure 3 shows SHAP values on the prediction level for the five folds used for CV, indicating the contribution of the RF input features (namely the probabilities of DR progression generated by all individual DCNNs) for the final prediction. Figure 3 generally confirms that high probabilities (dots in the red spectrum) for individual DCNNs contribute to the prediction of faster DR progression (appear on the positive part of the $x$-axis) and that low probabilities (dots in the blue spectrum) result in the opposite. This pattern is broken in the few cases where the aggregation used low- and highprobability values to predict both classes. This further confirms that using just one field would not be sufficient to properly address the prediction. In this particular case, Fig. 3 highlights that F5- and F7-specific DCNNs play a more crucial role in the prediction compared with F1 and F2.

\section{Attribution maps}

Attribution maps ${ }^{22-25}$ are a powerful strategy to shed light on the complex mechanism inside a single DCNN. These maps highlight areas where the model focuses its attention in order to decide how to classify a certain query image. Attribution maps are useful to check whether the decision mechanism of a DCNN is relatable to human expectation. The maps analyzed in this study were generated by guided back propagation, ${ }^{24}$ which provided the most salient results among different attribution techniques that were tested.

Figure 4 offers examples of different target time points and CFP fields, where test images are placed side-by-side to the corresponding attribution map. In general, the DCNNs seem to focus mainly on hemorrhages and microaneurysms, and partially on exudates, which are well recognized for their association with $D R{ }^{26} \mathrm{~A}$ remarkable aspect is that the DCNNs are able to highlight very small and low-contrast microaneurysms, even though they were not explicitly designed to accomplish this task. The correlation between microaneurysms and DR progression has been studied by Piri et al. ${ }^{27}$ and our attribution maps seem to be in line with these studies. However, there is an important difference; in our work, the DCNNs learn a functional mapping from baseline to month $X(6,12$, or 24$)$ using a single time point rather than the multiple visits used by Piri et al. ${ }^{27}$ to measure microaneurysm turnover. Please see the Supplement for examples of how attribution maps on the same image vary from month 6 through 24 (Supplementary Fig. 3) or with different repetitions of the prediction model (Supplementary Fig. 4).

Examples highlighted in Fig. 4 indicate that pathologies such as microaneurysms, hemorrhages, and hard exudates could be predictive for DR progression. Future studies may confirm this hypothesis by, for example, replacing $\mathrm{DL}$ with symbolic $\mathrm{ML}$ methods operating on hand-crafted features based on these pathologies.

\section{DISCUSSION}

This work demonstrates the feasibility of developing a DL algorithm to identify patients who will experience DR worsening by two or more ETDRS DRSS steps over the next 2 years, based solely on CFPs acquired at a single visit. Currently, it is only possible to approximate progression risk for groups of patients with similar signs and symptoms on the basis of their assessed DR severity level, but it is not possible to accurately predict the course of DR in an individual patient. ${ }^{20}$ Previous data from ETDRS studies demonstrated that DR is a slowly progressing disease and that there is an increasing risk of DR worsening and vision-threatening events as DR severity increases. ${ }^{20}$ For example, using the traditional manual grader assessment, a patient with moderately severe non-proliferative DR (NPDR) will have an $\sim 20 \%$ risk, whereas a patient with severe NPDR will have an $\sim 50 \%$ risk of progressing to proliferative DR (PDR) within 1 year. ${ }^{20}$ However, it is not know which individual patients belong to the subsets of fast versus slow progressors. This type of individual prediction can be achieved by means of the DL algorithms described in this paper.

A second crucial finding of this study was that any imagingbased diagnostic/predictive AI tool for DR should contemplate the inspection of both central and peripheral retina, instead of being limited to the use of CFPs centered around the fovea or optic nerve (F2, in the traditional ETDRS 7-field photography). ${ }^{15-19}$ Moreover, lateral fields may contain predictive signs of DR worsening before the pathology has affected the macula, allowing for prompt referral and, eventually, timely treatment before vision loss occurs. Coupling algorithms such as the one described here with more recent imaging technologies, such as ultrawide field (UWF) photography, might enable the identification of fast DR progressors even earlier in their disease course. The non-mydriatic UWF CFPs ${ }^{28-30}$ were recently validated for DR severity assessments and have the advantage of capturing a view of 200 degrees of the retina with less time and effort compared with the standard 7-field CFP, while providing good to excellent agreement in determining DR severity. ${ }^{29,30}$ Part of our algorithm validation and expansion strategy is to guarantee that the described algorithm will be capable of operating on UWF CFPs as well.

An inherent limitation of the present work is that our DCNNs have been developed and evaluated on two large, identically designed, sham-controlled, double-masked, phase 3 clinical trials (RIDE [NCT00473382] and RISE [NCT00473330]). ${ }^{31}$ The advantage of using clinical trial data is the availability of high-quality standardized imaging formats and processes as well as assessments by masked experts at a centralized reading center. However, this means that, at this time, our work is only applicable to clinical trial populations within a similar range of pre-specified eligibility criteria. Therefore, validation with datasets acquired in the real world will be essential to ensure that these results are reproducible and applicable to the broader DR population; the authors are already tackling this issue. The great advantage of the dataset at our disposal is that it contains a representation of 
Fold 0

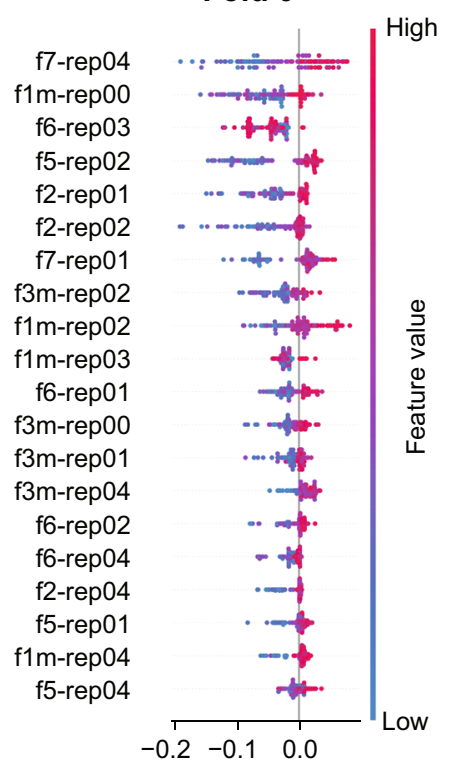

SHAP value (impact on model output)

\section{Fold 1}

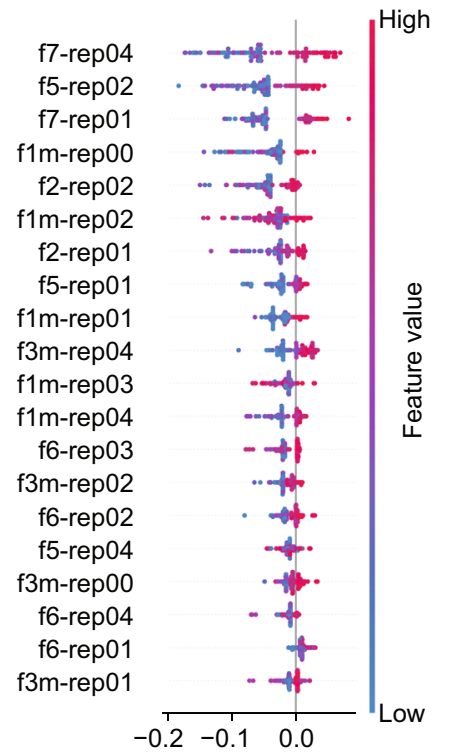

SHAP value (impact on model output)

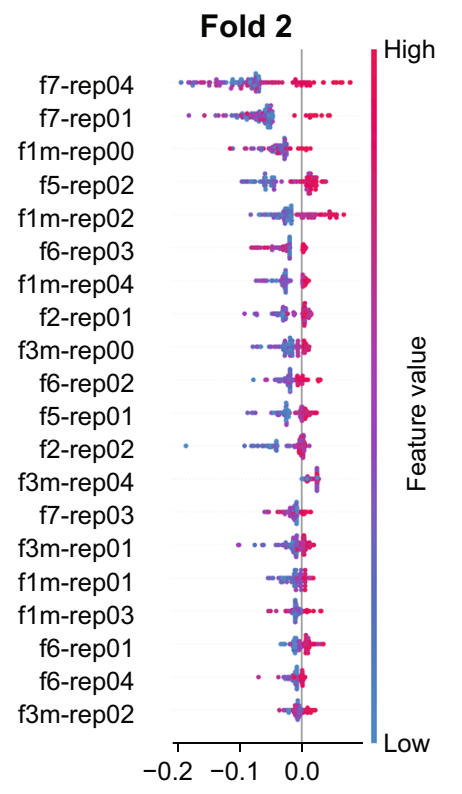

SHAP value (impact on model output)
Fold 3

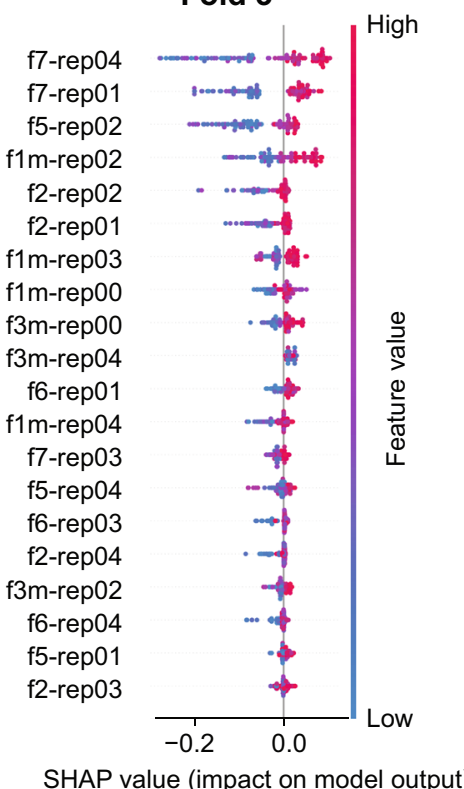

Fold 4

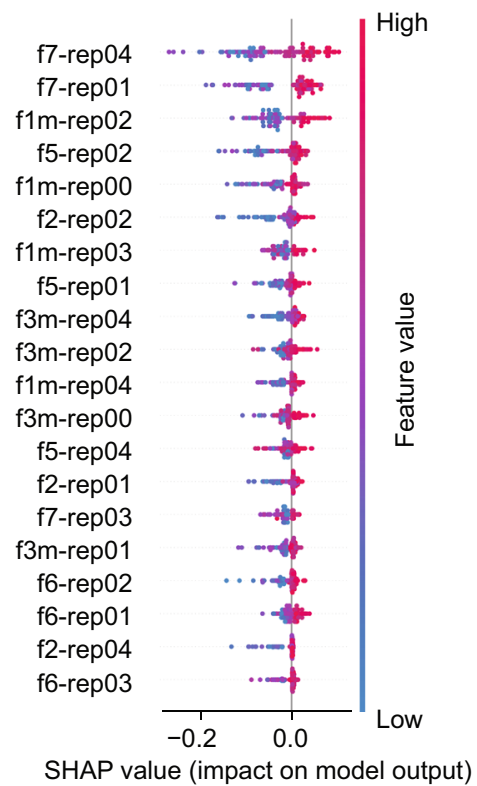

Fig. 3 SHAP plots summarizing the pointwise and average contribution of each deep convolutional neural network (DCNN) to the random forest aggregation. SHAP plots outlining the pointwise contribution of each DCNN. In this example, the SHAP analysis is related to the five folds used for the prediction of DR progression at month 24 is showed. The DCNNs are ordered in importance from top to bottom. The naming convention of the DCNNs highlights the field ('f1,' 'f2,' etc.) and repetition ('rep00,' 'rep01,' etc.)

patients across all levels of DR severity (see the distribution of RIDE/RISE patients with respect to the ETDRS DRSS overlaid with the number of 2-step DR progressors in Supplementary Fig. 1). This was key to testing the algorithm reliability across a diverse DR population. To summarize, the following aspects represent crucial limitations of the presented work: (a) relatively small patient population ( 530 patients); (b) patient population fulfilling the eligibility criteria of the RIDE and RISE clinical trials, thus not representing the real world population of patients with diabetes; (c) lack of an external validation set; and (d) limited interpretability of the overall prediction models due to the use of DCNN pillars to separately process CFP fields of view. Another potential limitation of the current analyses was the definition of DR progression. The definition we used is based on the clinically relevant magnitude of step-change on the ETDRS DRSS, which is not commonly used in the clinic. Although this scale was designed based on the correlation with increasing risk of vision loss and has been validated and accepted by regulators around the world, our next step is to explore the feasibility of an algorithm that predicts vision loss directly.

Our work represents a step forward in the application of Al to the field of ophthalmology. The DL modeling presented here differs from previously mentioned studies which either required multiple visits, did not employ $M L$, or focused on current DR severity diagnosis instead of prediction of the future. Additionally, as highlighted before, peripheral CFP fields have not been used in 
most of the previous $\mathrm{ML}$ and $\mathrm{DL}$ approaches dedicated to $\mathrm{DR}$ diagnostic development. ${ }^{15-19}$

Our findings suggest that deployment of a predictive DR progression algorithm would enable early identification of patients at highest risk of vision loss, allowing timely referral to retina specialists and potential initiation of treatment before irreversible vision loss occurs. In the context of the global diabetes epidemic, new screening tools could have a substantial socioeconomic impact on the healthcare system. The federal savings are projected to reach $\$ 624.0$ million annually in the United States alone, if we can deploy tools that enable recommended eye screening and ophthalmic care for all diabetes patients. ${ }^{32}$ However, these estimates are based on the ability to expand diagnosis across members of the diabetes population currently lacking access to medical care. To our knowledge, nobody has yet performed an evaluation of cost savings based on predictive screening, which would identify individuals who require immediate follow up and possible early intervention due to impeding near-future progression. Deployment of a predictive algorithm represents therefore an important conceptual leap for the efficient triage of patients at high risk of vision-threatening DR complications and a step towards a personalized approach to medicine, what it also envisaged as "precision medicine."

Moreover, the identification of fast DR-progressing patients through a predictive DR progression algorithm would have the potential to support the development of new treatments targeting patients with mild and moderate NPDR. Clinical trials based on the traditional endpoint of DR improvement/worsening are considered prohibitively expensive due to size and/or duration. By enriching the clinical trial population with fast DRprogressing individuals, such an Al-based recruitment strategy would increase the chances of success for clinical trials of novel drugs designed to address the unmet need of those members of the early DR population at the greatest risk of progression and vision loss. This is particularly important considering the rising global prevalence of DR and its potential impact on healthcare systems and society.

\section{METHODS}

\section{Dataset}

This study is based on the post hoc retrospective analysis of stereoscopic 7-field CFPs obtained from eyes with DR in the RIDE (NCT00473382) ) $^{33-35}$ and RISE (NCT00473330) (3-35 $^{33}$ phase 3 studies at baseline (start of the studies) that were not treated with anti-vascular endothelial growth factor (VEGF) therapy. RIDE and RISE were registered on 13/05/2007 with the title "A Study of Ranibizumab Injection in Subjects With Clinically Significant Macular Edema (ME) With Center Involvement Secondary to Diabetes Mellitus" and can be accessed via the ICTRP portal at the following URLs: RIDE at http://apps.who.int/trialsearch/Trial2.aspx?TriallD $=$ NCT00473382, RISE at http://apps.who.int/trialsearch/Trial2.aspx?TrialID $=$ NCT00473330.

The objective of this analyses was to generate algorithms that can predict worsening in untreated eyes from baseline over a period of 2 years. From RIDE and RISE, only the baseline images from those eyes that were randomized to sham/untreated groups and the untreated fellow eyes were used for this work. These eyes had the natural course of DR worsening outcomes without anti-VEGF treatment collected at months 6,12 , and 24 . There was a total of 529 (683 eyes, 4781 CFPs), 528 (682 eyes, 4774 CFPs), and 499 (645 eyes, 4515 CFPs) patients with untreated eyes who had all seven fields captured on CFP at months 6,12 , and 24. RIDE and RISE were two parallel, identically designed, phase 3 , randomized, double-masked clinical trials of ranibizumab in patients with DR with diabetic macular edema. The studies were sham injection controlled for 2 years and followed for an additional year in which all sham patients crossed over to ranibizumab therapy. The study design, eligibility criteria, and core efficacy and safety outcomes of these trials have been previously described. ${ }^{32-34}$ Baseline ETDRS DRSS DR severity in RIDE/RISE sham-treated study and fellow eyes ranged from 10 (absent) to 71 (high-risk PDR).$^{20}$ The manually detected rates of two-step or more worsening in sham study eyes and fellow eyes at 2 years from baseline were $9.6 \%$ and $11.7 \%$, respectively. ${ }^{33}$ a

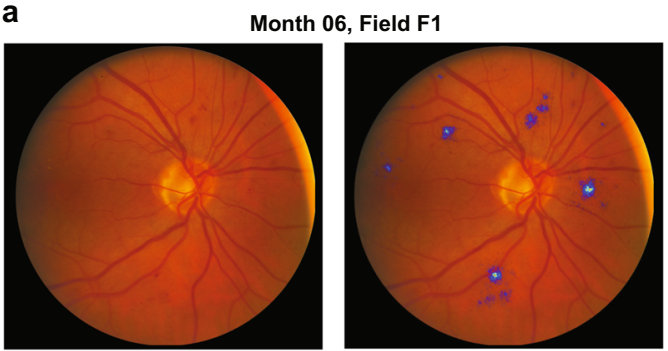

Month 06, Field F2
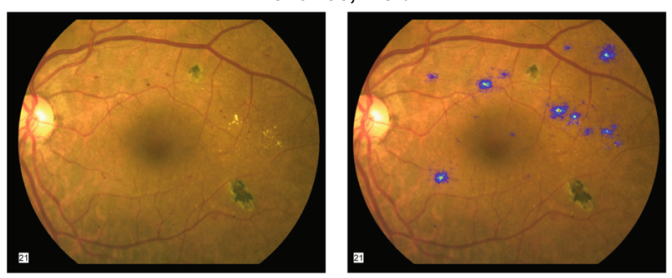

b

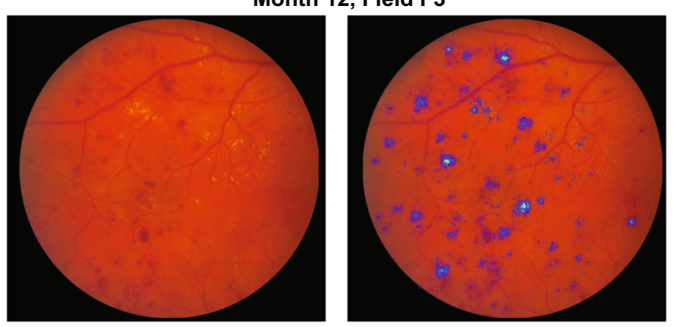

Month 12, Field F7

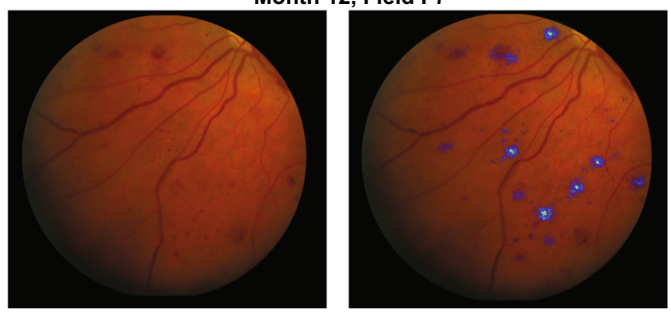

C

Month 24, Field F4

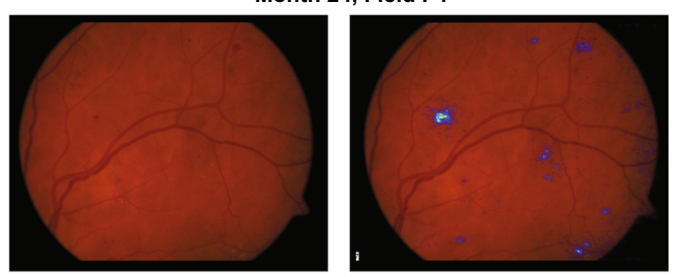

Month 24, Field F6
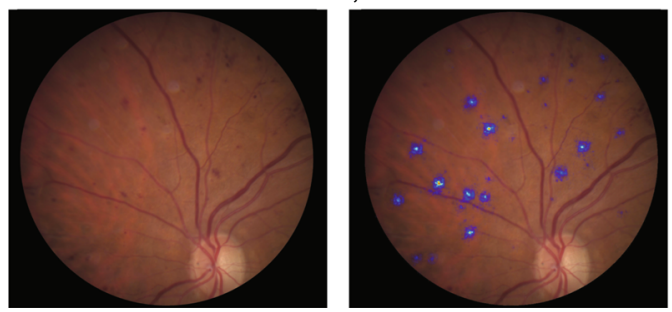

The majority of the CFP images were of high quality due to the training requirement for all study site photographers who participated in CFP acquisition. Additionally, the image assessment for the manual severity grading was of the highest attainable quality because it was performed by two masked readers at an accredited fundus photograph reading center supervised by a senior reader in charge of adjudication when needed. 
Fig. 4 Example of attribution maps placed side by side to the original test color fundus image. In each set, the original image is on the left and the attribution map is on the right. The attribution of the deep convolutional neural networks focuses mainly on microaneurysms, hemorrhages, and exudates. a Two examples of attribution maps for the model predicting diabetic retinopathy (DR) progression at month 6 . $\mathbf{b}$ Two examples of attribution maps for the artificial intelligence model predicting DR progression at month 12. c Two examples of attribution maps for the model predicting DR progression at month 24

Each patient data point at each visit consists of seven CFP fields that span a 30-degree view of retina. The different fields are indicated with the following codes: F1 (optic nerve centered); F2 (fovea centered); and F3, F4, F5, F6, and F7 (all peripheral fields); all codes correspond to a general ETDRS standard adopted by reading centers. ${ }^{35}$

The trials adhered to the tenets of the Declaration of Helsinki and were Health Insurance Portability and Accountability Act compliant, and the protocols were approved by the study sites' institutional review boards and ethics committees (or as applicable). Patients provided written informed consent.

\section{Outcome variable for DR progression}

The 7-field CFPs acquired for each patient at baseline were used to train DL models designed to predict, on an individual patient level, two-step or more worsening with respect to the ETDRS DRSS over 2 years, specifically after 6,12 , and 24 months. The problem under study is a binary classification, where ' 0 ' means no occurrence of two-step or more ETDRS DRSS worsening at month $X(6,12$, or 24$)$, and ' 1 ' means the opposite. The incidence of sham study and fellow eyes diagnosed with worsening by two or more ETDRS DRSS steps was $\sim 6 \%$ at month $6, \sim 10 \%$ at month 12 , and $\sim 12 \%$ at month 24 . The histograms in Supplementary Fig. 1 show the distribution of the population of sham study and fellow eyes at baseline with respect to the ETDRS DRSS overlaid with the number of DR progressors and non-progressors for month 6, 12, and 24 .

The ETDRS DRSS scale has been validated and widely used for objective quantification of retinopathy severity in the clinical trial setting. The landmark trials, Diabetic Retinopathy Study and ETDRS, established that worsening of DR (that is measured by anatomic features on the ETDRS DRSS) is significantly associated with development of severe vision loss. ${ }^{36}$ Moreover, the incidence of clinically significant diabetic macular edema was shown to correlate with the progression of DR on the ETDRS DRSS from NPDR to PDR. ${ }^{20}$ Even just one-step or more DRSS worsening was shown to be associated with a five- to sixfold increased risk of PDR development, and a three- to fourfold risk of developing clinically significant macular edema with a high likelihood of vision loss over the period of 4 years. ${ }^{37}$ However, considering the intergrader variability associated with this scale, two steps or more on the ETDRS DRSS was deemed a more robust outcome variable to use for predictive modeling.

\section{Modeling}

The overall model is a binary classification whose input data points are sets of seven CFP images acquired for a selected eye at baseline. Modeling was performed in two steps: (1) DCNNs were separately trained for each type of CFP field to form the "pillars"; and (2) the probabilities provided by the individual pillars are then aggregated by means of RFs. Single pillars and $\mathrm{RFs}$ are trained against the binary outcome variable defined in the previous section. A simple schematic of the model is provided by Fig. 1c.

The Inception-v3 architecture ${ }^{38}$ was used to build the field-specific pillars. A transfer learning ${ }^{39}$ cascade strategy was adopted to create the starting weights; first, the architecture was initialized with Imagenet ${ }^{40}$ weights and trained on the Kaggle $\mathrm{DR}^{41}$ dataset to differentiate between CFPs with no DR from those with signs of DR; the weights generated by this last training are then used to initialize the training of the pillars for DR progression.

Transfer learning was performed, first by replacing and training for 10 epochs the last dense layers while keeping all the others fixed, and then by fine-tuning for 50 epochs all layers from the end up to the third one. The Adam optimizer was used with learning rates adapted for the various pillars. A small parameter grid search was conducted to find the optimal learning rates for the pillars. A five-times fivefold CV scheme with patient
ID constraint (data points of the eyes of the same patient were constrained to belong to the same $\mathrm{CV}$ fold; i.e., both either to the training or testing set) was adopted to generate a total of 25 DCNNs for each pillar.

$\mathrm{RFs}^{42}$ were then used to aggregate the probabilities of all pillars. A fivefold CV scheme with the same folds of the previous step was performed. This means that for each fold, 35 probabilities (seven fields $\times$ five repetitions for each CV fold) were used as input features for the RF. Please see Supplementary Methods for additional information about the RF models used in this study.

The model performance was measured in terms of AUC, sensitivity, and specificity evaluated at the Youden's point, ${ }^{43}$ which is obtained by maximizing the difference between the true- and false-positive rate.

SHAP is a technique originally adopted in game theory to determine how much each player in a collaborative game has contributed to its success. In the ML context, each SHAP value measures how much each feature contributes to the target prediction, either in a positive or a negative way. ${ }^{21}$ The traditional feature importance algorithm is based on the Gini index highlights, which features are contributing the most to the prediction across the entire population ${ }^{44}$ and is known in literature to be characterized by multiple biases, ${ }^{45}$ preventing this algorithm to be reliable in many scenarios. Differently from the Gini index, SHAP offers a local perspective by informing on the most predictive features at the level of individual sample. ${ }^{21}$ In the plots of Fig. 3, each row corresponds to an input feature and each colored dot is a sample, whose color ranges from blue to red to inform whether the selected input feature has a low or a high value for the selected sample.

The attribution maps for the individual DL models presented in Fig. 4 were generated by means of a gradient-based technique called guidedbackpropagation. ${ }^{46}$ The idea behind gradient-based methods is that the relative importance of the input features is measured by computing the gradient of the output decision with respect to those input features. ${ }^{47}$ This gradient, once back-projected onto the input image, provides an idea of where the CNN has focused on in order to classify the image in a certain way. In particular, guided-backpropagation ${ }^{46}$ is characterized by the suppression of flow of gradients where either the input or incoming gradients are negative.

\section{Reporting summary}

Further information on research design is available in the Nature Research Reporting Summary.

\section{DATA AVAILABILITY}

Qualified researchers may request access to individual patient level data through the clinical study data request platform (www.clinicalstudydatarequest.com). Further details on Roche's criteria for eligible studies are available here (https:// clinicalstudydatarequest.com/Study-Sponsors/Study-Sponsors-Roche.aspx). For further details on Roche's Global Policy on the Sharing of Clinical Information and how to request access to related clinical study documents, see here (https://www. roche.com/research_and_development/who_we_are_how_we_work/clinical_trials/ our_commitment_to_data_sharing.htm).

\section{CODE AVAILABILITY}

The code to create the FOV-specific CNN pillars is based on Keras using Tensorflow as backend and is available at this public Github repository (https://github.com/arcaduf/ nature_paper_predicting_dr_progression). The repository contains the YAML configuration files used to select the training hyper-parameters of each FOV-specific CNN pillar and a detailed README file outlining the content of the folders and how to make use of the scripts.

Received: 15 April 2019; Accepted: 3 September 2019; Published online: 20 September 2019

\section{REFERENCES}

1. International Diabetes Federation. IDF diabetes atlas: 8th edn. http://www. diabetesatlas.org/ (2017).

2. Fong, D. S. et al. American Diabetes Association. Retinopathy in diabetes. Diabetes Care 27, S84-S87 (2004).

3. Brar, M. \& Ho, A. C. Diabetic eye disease: a multidisciplinary discussion on managing diabetic eye disease. EyeNet Magazine Suppl. 1-4 (2016). 
4. Murchison, A. P. et al. Nonadherence to eye care in people with diabetes. BMJ Open Diab. Res. Care 5, e000333 (2017).

5. Mazhar, K. et al. Los Angeles Latino Eye Study Group Severity of diabetic retinopathy and health-related quality of life: the Los Angeles Latino Eye Study. Ophthalmology 118, 649-655 (2011).

6. National Center for Chronic Disease Prevention Health Promotion; Division of Diabetes Translation. National Diabetes Statistics Report. https://stacks.cdc.gov/ view/cdc/23442 (2014).

7. Willis, J. R. et al. Vision-related functional burden of diabetic retinopathy across severity levels in the United States. JAMA Ophthalmol. 135, 926-932 (2017).

8. Krizhevsky, A., Sutskever, I. \& Hinton, G. E. Imagenet classification with deep convolutional neural networks. Adv. Neural Inf. Process. Syst. https://papers.nips.cc/paper/ 4824-imagenet-classification-with-deep-convolutional-neural-networks (2012).

9. Yu, K.-H., Beam, A. L. \& Kohane, I. S. Artificial intelligence in healthcare. Nat. Biomed. Eng. 2, 719-731 (2018).

10. Marr, B. First FDA approval for clinical cloud-based deep learning in healthcare. Forbes. https://www.forbes.com/sites/bernardmarr/2017/01/20/first-fda-approvalfor-clinical-cloud-based-deep-learning-in-healthcare/\#112785c9161c (2017).

11. Samala, R. K. et al. Mass detection in digital breast tomosynthesis: deep convolutional neural network with transfer learning from mammography. Med. Phys. 43, 6654-6666 (2016).

12. Lakhani, P. \& Sundaram, B. Deep learning at chest radiography: automated classification of pulmonary tuberculosis by using convolutional neural networks. Radiology 284, 574-582 (2017).

13. Esteva, A. et al. Dermatologist-level classification of skin cancer with deep neural networks. Nature 542, 115-118 (2017).

14. Litjens, G. et al. A survey on deep learning in medical image analysis. Med. Image Anal. 42, 60-88 (2017).

15. Gulshan, V. et al. Development and validation of a deep learning algorithm for detection of diabetic retinopathy in retinal fundus photographs. JAMA 316, 2402-2410 (2016).

16. Ting, D. S. W. et al. Development and validation of a deep learning system for diabetic retinopathy and related eye diseases using retinal images from multiethnic populations with diabetes. JAMA 318, 2211-2223 (2017).

17. U.S. Food and Drug Administration. FDA permits marketing of artificial intelligence-based device to detect certain diabetes-related eye problems. https://www.fda.gov/newsevents/newsroom/pressannouncements/ucm604357. htm (2018).

18. Poplin, R. et al. Prediction of cardiovascular risk factors from retinal fundus photographs via deep learning. Nat. Biomed. Eng. 2, 158-164 (2018).

19. Arcadu, F. et al. Deep learning predicts OCT measures of diabetic macular thickening from color fundus photographs. Invest. Ophthalmol. Vis. Sci. 60, 852-857 (2019).

20. Early Treatment Diabetic Retinopathy Study Research Group. Grading diabetic retinopathy from stereoscopic color fundus photographs-an extension of the modified Airlie House classification: ETDRS report number 12. Ophthalmology $\mathbf{9 8}$, 823-833 (1991).

21. Lundberg, S. M. \& Lee, S. I. A unified approach to interpreting model predictions. Adv. Neural Inf. Process. Syst. https://papers.nips.cc/paper/7062-a-unifiedapproach-to-interpreting-model-predictions (2017).

22. Simonyan, K., Vedaldi, A. \& Zisserman, A. Deep inside convolutional networks: visualising image classification models and saliency maps. https://arxiv.org/abs/ 1312.6034 (2014).

23. Zeiler M.D., Fergus R. in Computer Vision - ECCV 2014. ECCV 2014. Lecture Notes in Computer Science, vol 8689 (eds Fleet, D. et al.) (Springer, Cham, 2014).

24. Springenberg, J. T., Dosovitskiy, A., Brox, T. \& Riedmiller, M. Striving for simplicity: the all convolutional net. https://arxiv.org/abs/1412.6806 (2015).

25. Samek, W., Binder, A., Montavon, G., Lapuschkin, S. \& Müller, K. R. Evaluating the visualization of what a deep neural network has learned. IEEE Trans. Neural Netw. Learn. Syst. 28, 2660-2673 (2017).

26. Pappuru, R. K. R., Ribeiro, L., Lobo, C., Alves, D. \& Cunha-Vaz, J. Microaneurysm turnover is a predictor of diabetic retinopathy progression. Br. J. Ophthalmol. 103, 222-226 (2019).

27. Piri, S., Delen, D., Liu, T. \& Zolbanin, H. M. A data analytics approach to building a clinical decision support system for diabetic retinopathy: developing and deploying a model ensemble. Dec. Support Syst. 101, 12-27 (2017).

28. Silva, S. P. et al. Hemorrhage and/or microaneurysm severity and count in ultrawide field images and Early Treatment Diabetic Retinopathy Study photography. Ophthalmology 124, 970-976 (2017).

29. Ghasemi Falavarjani, K., Tsui, I. \& Sadda, S. R. Ultra-wide-field imaging in diabetic retinopathy. Vis. Res. 139, 187-190 (2017).

30. Aiello, L. P. et al. Comparison of Early Treatment Diabetic Retinopathy Study standard 7-field imaging with ultrawide-field imaging for determining severity of diabetic retinopathy. JAMA Ophthalmol. 137, 65-73 (2019).
31. Brown, D. M. et al. Long-term outcomes of ranibizumab therapy for diabetic macular edema: the 36-month results from two phase III trials: RISE and RIDE. Ophthalmology 120, 2013-2022 (2013).

32. Javitt, J. C. et al. Preventive eye care in people with diabetes is cost saving to the federal government. Implic. Health Care Reform. Diabetes Care 17, 909-917 (1994).

33. Ip, M. S., Domalpally, A., Hopkins, J. J., Wong, P. \& Ehrlich, J. S. Long-term effects of ranibizumab on diabetic retinopathy severity and progression. Arch. Ophthalmol. 130, 1145-1152 (2012).

34. Moss, S. E., Klein, R. \& Klein, B. E. The 14-year incidence of visual loss in a diabetic population. Ophthalmology 105, 998-1003 (1998).

35. Brown, D. M. et al. Long-term outcomes of ranibizumab therapy for diabetic macular edema: the 36-month results from two phase III trials: RISE and RIDE. Ophthalmology 120, 2013-2022 (2013).

36. Royle, P. et al. Pan-retinal photocoagulation and other forms of laser treatment and drug therapies for non-proliferative diabetic retinopathy: systematic review and economic evaluation. Health Technol. Assess. 19, 1-247 (2015).

37. Klein, R., Klein, B. E. K. \& Moss, A. E. How many steps of progression of diabetic retinopathy are meaningful? Arch. Ophthalmol. 119, 547-553 (2001).

38. Szegedy, C., Vanhoucke, V., loffe, S., Shlens, J. \& Wojna, Z. Rethinking the inception architecture for computer vision. https://arxiv.org/abs/1512.00567 (2015).

39. Yosinski, J., Clune, J., Bengio, Y. \& Lipson, H. How transferable are features in deep neural networks? Adv. Neural Inf. Process. Syst. https://papers.nips.cc/paper/5347how-transferable-are-features-in-deep-neural-networks (2014).

40. Deng, J. et al. ImageNet: a large-scale hierarchical image database. CVPR. http:// www.image-net.org/papers/imagenet_cvpr09.pdf (2009).

41. Kaggle. Diabetic retinopathy detection. https://www.kaggle.com/c/diabeticretinopathy-detection (2017).

42. Breiman, L. Random forests. J. Mach. Learn. 45, 5-32 (2001).

43. Youden, W. J. Index for rating diagnostic tests. Cancer 3, 32-35 (1950).

44. Breiman, L., Friedman, J., Stone, C. J. \& Olshen, R. A. Classification and Regression Trees. (CRC Press, Boca Raton, 1984).

45. Strobl, C., Boulesteix, A. L., Zeileis, A. \& Hothorn, T. Bias in random forest variable importance measures: illustrations, sources and a solution. BMC Bioinforma. 8, 25 (2007).

46. Springenberg, J. T., Dosovitskiy, A., Brox, T. \& Riedmiller, M. Striving for simplicity: the all convolutional net. https://arxiv.org/abs/1412.6806 (2015).

47. Simonyan, K., Vedaldi, A. \& Zisserman, A. Deep inside convolutional networks: visualising image classification models and saliency maps. https://arxiv.org/abs/ 1312.6034 (2014).

\section{ACKNOWLEDGEMENTS}

Funding was provided by Roche Inc. and Genentech, Inc., a member of the Roche Group. Genentech, Inc., provided support for the study and contributed to the study design, study conduct, data collection, management, and interpretation. We would also like to express gratitude to Anthony Adamis, MD, for his visionary leadership and the profound impact he has had as the champion of innovation and personalized healthcare in ophthalmology at Genentech-Roche, attracting experts from across relevant fields of expertise to create a fruitful environment that is conducive of cutting edge research to help patients with high unmet need.

\section{AUTHOR CONTRIBUTIONS}

All authors had full access to the data and contributed to the design of the study and to the data interpretation, preparation, writing, and approval of the manuscript. F.A. and Z.H. participated in data collection, management, and analyses.

\section{COMPETING INTERESTS}

F.A., F.B., M.P., and A.M. are employees and shareholders of Roche, Inc. J.W. and Z.H. are employees and shareholders of Genentech, Inc.

\section{ADDITIONAL INFORMATION}

Supplementary information is available for this paper at https://doi.org/10.1038/ s41746-019-0172-3.

Correspondence and requests for materials should be addressed to Z.H. or M.P.

Reprints and permission information is available at http://www.nature.com/ reprints 
Publisher's note Springer Nature remains neutral with regard to jurisdictional claims in published maps and institutional affiliations.

cc (i) Open Access This article is licensed under a Creative Commons Attribution 4.0 International License, which permits use, sharing, adaptation, distribution and reproduction in any medium or format, as long as you give appropriate credit to the original author(s) and the source, provide a link to the Creative
Commons license, and indicate if changes were made. The images or other third party material in this article are included in the article's Creative Commons license, unless indicated otherwise in a credit line to the material. If material is not included in the article's Creative Commons license and your intended use is not permitted by statutory regulation or exceeds the permitted use, you will need to obtain permission directly from the copyright holder. To view a copy of this license, visit http://creativecommons. org/licenses/by/4.0/.

(c) The Author(s) 2019, corrected publication 2020 